Mise au point

\title{
Préparation physique et système respiratoire : les limites de l'adaptation
}

\section{Physical preparation and respiratory system: The limits of the adaptation}

\author{
${ }_{4} \mathrm{Q} 1 \mathrm{~T}_{\wedge}^{\mathrm{T}}$. Bury ${ }_{\wedge}^{*}, \mathrm{~S}_{\text {. Hody }}$ \\ Département des sciences de la motricité, université de Liège, quartier Blanc-Gravier, bâtiment B21, allée des Sports 2, 4000 Liège Sart Tilman, Belgique
}

\section{N F O A A R T I C L E}

\section{Historique de l'article :}

Disponible sur Internet le xxx

\section{Mots clés :}

Exercice

Fonction pulmonaire

Hypoxémie

Asthme

\section{Keywords:}

Exercise

Respiratory system

Hypoxemia

Asthma

\begin{abstract}
R É S U M É
L'aptitude à maintenir un haut niveau d'activité sans fatigue dépend, d'une part, d'un bon fonctionnement des systèmes d'approvisionnement en $\mathrm{O}_{2}$ et, d'autre part, de la capacité des cellules musculaires à produire de l'ATP en aérobiose. Nous décrivons, dans cet article, les réponses aiguës et chroniques du système respiratoire à l'exercice ainsi que les éventuelles limites d'adaptation de ce système au cours d'une préparation physique. Nous abordons, également, de façon succincte, l'intérêt d'un entraînement spécifique du système respiratoire.
\end{abstract}

(c) 2018 Publié par Elsevier Masson SAS.

\section{A B S T R A C T}

The ability to sustain a high level of physical activity without undue fatigue depends on the capacity of the physiologic systems for oxygen delivery and on the capacity of the muscle cells to generate ATP aerobically. In this article, we describe respiratory responses to acute and chronic exercise. We discuss respiratory limitations to performance and effects of respiratory muscle training on performance in athletes.

() 2018 Published by Elsevier Masson SAS.

\section{Introduction}

L'augmentation de la dépense énergétique aérobie à l'exercice nécessite des ajustements, en particulier des systèmes respiratoire et cardiovasculaire. Le principal objectif de ces ajustements est d'assurer un approvisionnement correct en oxygène aux tissus en activité afin de permettre une production accrue d'ATP dont le rôle est essentiel dans la contraction musculaire.

Dans cet article, nous présentons, de façon succincte, les réponses ainsi que les limites du système respiratoire lors de l'effort physique chez le sportif.

\footnotetext{
* Auteur correspondant.

Adresse e-mail : tbury@uliege.be (T. Bury).
}

\section{Ajustements respiratoires à l'exercice}

Jusqu'à une intensité modérée d'efforts physiques, le débit ventilatoire augmente proportionnellement à la consommation d'oxygène et à la production de gaz carbonique ; il est de 20 à 25 litres d'air par litre d'oxygène consommé. À ces intensités, le débit ventilatoire croit, principalement par l'augmentation du volume courant, alors qu'à plus haute intensité, la fréquence respiratoire joue un rôle plus important. Le temps de transit du sang dans les capillaires pulmonaires étant suffisamment long, malgré l'augmentation du débit cardiaque, l'équilibre des gaz à travers la membrane alvéolo-capillaire se réalise.

À des niveaux d'exercices plus intenses, le débit ventilatoire s'accroît de façon disproportionnée par rapport à la consommation d'oxygène. L'équivalent ventilatoire augmente donc et peut atteindre 35 à 45 litres d'air par litre d'oxygène consommé. La puissance au changement de pente de la ventilation (selon les sujets et leur niveau d'aptitude, entre 60 et $90 \%$ de la $\mathrm{VO}_{2} \max$ ) correspond au seuil ventilatoire, lequel est généralement contemporain du 
seuil d'augmentation du lactate sanguin ou seuil anaérobie. Il représente un bon indice de l'aptitude du sportif à l'exercice prolongé. Le débit ventilatoire qui, au repos, chez l'adulte, est de 5 à $8 \mathrm{~L} / \mathrm{min}$, peut atteindre, lors de l'exercice maximal, des valeurs de 100 à $120 \mathrm{~L} / \mathrm{min}$ chez les sujets modérément entraînés et de 150 à $200 \mathrm{~L} / \mathrm{min}$ chez les athlètes de haut niveau. Les observations faites sur les sportifs de bon niveau montrent que ces derniers adoptent spontanément un régime ventilatoire avec un volume courant important et une fréquence respiratoire basse. Il est admis que ce régime correspond généralement à une efficacité ventilatoire optimale avec une dépense minimale d'énergie par les muscles respiratoires $[1,2]$. Cependant, dans de nombreux types d'exercices, le régime ventilatoire est dépendant du rythme des mouvements.

Il existe également, au cours d'un exercice, des ajustements au niveau de l'échangeur respiratoire. En effet, le rapport ventilation/perfusion se modifie considérablement à l'exercice. La ventilation augmente environ 5 fois plus que la perfusion. Ce rapport optimal n'est plus de 1 , comme au repos, mais de 3 . Il y a donc une prédominance des hauts rapports ventilation/perfusion, favorables à une bonne hématose.

Les fortes augmentations de la ventilation pulmonaire à l'exercice n'ont pratiquement pas d'effets sur le niveau de résistance des voies aériennes. Cela s'explique par une respiration souvent buccale qui supprime la contribution des voies aériennes supérieures, normalement élevée au repos, et par une dilatation laryngée. De la sorte, les pressions mesurées au niveau de l'arbre bronchique restent relativement faibles lors des efforts physiques, même de type maximaux [1].

Sur la base de ces différentes observations, il est généralement admis dans la littérature que la ventilation pulmonaire ne constitue pas, dans les conditions normales et chez le sportif sain, un facteur limitant l'aptitude du sportif à effectuer un exercice physique de type aérobie. Ceci est notamment illustré par le fait que la consommation maximale d'oxygène est atteinte alors que la ventilation peut encore s'accroître, soit en augmentant la puissance d'exercice, soit en demandant au sujet d'hyper ventiler volontairement. Enfin, si le travail ventilatoire augmente par rapport à sa valeur de repos, l'énergie supplémentaire que cela exige reste faible comparativement à la dépense énergétique totale. Chez l'athlète de haut niveau cependant, la littérature scientifique tend à admettre que l'exercice pourrait être limité par la ventilation notamment parce que le débit ventilatoire maximal d'exercice peut atteindre des valeurs égales à la ventilation maximale volontaire possible.

\section{Adaptations respiratoires à l'entraînement}

L'augmentation de la consommation maximale d'oxygène, consécutive à un entraînement à l'exercice intense et prolongé, est concomitante d'une meilleure adaptation des systèmes respiratoire, cardiovasculaire et musculaire à l'exercice.

À l'entraînement aérobie, les possibilités fonctionnelles ventilatoires sont améliorées. Cela peut déjà être manifeste au repos lors des épreuves de ventilation forcée : le débit ventilatoire instantané de pointe ainsi que la ventilation maximale par minute sont augmentés. Au repos, l'équivalent respiratoire n'est cependant pratiquement pas modifié. Lors de l'exercice sous-maximal, à métabolisme égal, l'individu entraîné a un débit ventilatoire, et par conséquent un équivalent respiratoire, plus bas. Cette diminution du niveau ventilatoire d'exercice est à rapprocher de la diminution du rôle de certains stimuli de contrôle ventilatoire tels que $\mathrm{O}_{2}$, $\mathrm{CO}_{2}$ et $\mathrm{H}^{+}$.

Après entraînement aérobie, le sportif respire donc moins d'air pour une quantité sous-maximale d'oxygène donnée, réduisant ainsi le coût énergétique de la respiration. Théoriquement, cette adaptation est importante au cours d'un exercice d'endurance soutenu car non seulement les muscles respiratoires se fatiguent moins vite mais, en outre, l'oxygène non utilisé par ces muscles demeure disponible pour les muscles au travail $[3,4]$.

Lors de l'exercice maximal, la ventilation est supérieure à celle observée avant entraînement ; il existe d'ailleurs, chez l'athlète, une relation étroite entre la $\mathrm{VO}_{2}$ max et le niveau de ventilation maximale. Cette observation est physiologiquement logique car une augmentation de la capacité aérobie signifie un plus grand besoin d'oxygène et une production accrue de gaz carbonique que l'on peut satisfaire par une augmentation du débit ventilatoire.

\section{Limites du système respiratoire au cours de la préparation physique en endurance}

\subsection{Hypoxémie induite par l'exercice (HIE)}

Chez les athlètes d'endurance de haut niveau, le système pulmonaire peut être pleinement sollicité, voire dépassé par la capacité fonctionnelle des autres "systèmes aérobies ", perturbant les échanges alvéolo-capillaires. On peut effectivement observer, chez ce type d'athlète s'exerçant à un niveau proche de leur $\mathrm{VO}_{2}$ max, une diminution de la quantité d'oxygène dans le sang artériel ainsi qu'une réduction de la saturation en oxygène du sang artériel. Pour poser ce diagnostic d'HIE, la chute de la $\mathrm{PaO}_{2}$ doit être d'au moins 10 millimètres de mercure ou celle de la $\mathrm{SaO}_{2}$ d'au moins $4 \%$. Cette anomalie gazométrique au cours de l'exercice chez l'athlète peut être identifiée de manière directe par prélèvement artériel (laboratoire) ou de manière indirecte à l'aide d'un saturomètre ou oxymètre de pouls. Il s'agit d'un petit appareil portable non invasif et donc utilisable sur le terrain. Dans la littérature, la relation entre HIE et intensité de l'effort est claire. L'influence de l'entraînement et du niveau de spécialisation dans la préparation physique en endurance sont également des facteurs qui potentialisent l'HIE. On considère que cette anomalie est observée chez quasi $50 \%$ des athlètes qui ont une $\mathrm{VO}_{2}$ max supérieure à $57 \mathrm{~mL} / \mathrm{kg} / \mathrm{min}$ et qui ont suivi pendant plusieurs années des entraînements d'endurance intense [5,6]. Les sports les plus touchés par ce phénomène sont les sports à forte dominante aérobie, en particulier s'ils sollicitent les membres supérieurs tels que le ski de fond, le triathlon et le cyclisme... La physiopathologie de cette anomalie reste discutée dans la littérature et associe une hypoventilation alvéolaire et la formation d'un œdème péribronchique mais aussi interstitiel. Cet œdème serait à la base d'un trouble de la diffusion alvéolo-capillaire. Indépendamment de l'intérêt physiopathologique, la question essentielle pour le médecin du sport ainsi que le sportif est de s'interroger sur le retentissement de cette HIE sur les performances [7]. À court terme, le risque principal est celui d'une limitation de la performance aérobie. À moyen et long termes, les répercussions de ces hypoxémies sur les principales fonctions physiologiques sont mal connues mais elles pourraient être impliquées dans le développement tardif d'une hypertension artérielle pulmonaire, voire être un des facteurs responsables de la mort subite des anciens athlètes. En pratique, chez un sportif de plus de 50 ans, coureur de fond ou cyclotouriste par exemple, il est conseillé d'éviter les intensités extrêmes et de limiter le niveau de l'activité habituelle à celui du seuil ventilatoire.

\subsection{Les troubles ventilatoires obstructifs}

Plusieurs enquêtes épidémiologiques ont constaté une prévalence anormalement élevée de symptômes d'asthme chez les athlètes pratiquant des sports en compétition $[8,9]$. Lorsqu'on analyse ces enquêtes épidémiologiques, on se rend compte que la fréquence de l'asthme varie en fonction du niveau de pratique mais également en fonction de la discipline sportive 
considérée. Ce sont essentiellement les athlètes de niveau international, se soumettant à un volume d'entrainement important (plus de 20 heures par semaine) depuis plusieurs années qui sont confrontés à ces symptômes bronchiques (toux, expectorations, sifflements, dyspnée...). Ceux-ci sont particulièrement fréquents dans les disciplines d'endurance. Ainsi, on note une prévalence de l'ordre de 25-40 \% d'athlètes catalogués d'asthmatiques chez les cyclistes et les marathoniens contre 5-7\% au sein de la population générale. C'est également vrai pour d'autres sports d'endurance, en particulier ceux qui sont réalisés dans des conditions environnementales irritatives pour le système bronchopulmonaire, comme le ski de fond (inhalation d'un air froid et sec) ou la natation de haut niveau (inhalation de dérivés chlorés). Pour expliquer cette inflammation et réactivité bronchique majorée chez ces athlètes d'endurance, la littérature scientifique incrimine essentiellement l'hyperventilation d'effort prolongée (air froid et sec, chargé en allergènes ou polluants en tout genre), susceptible d'assécher et de refroidir les voies aériennes. La plupart des athlètes qui en sont victimes ne s'en inquiètent guère alors que ces anomalies bronchiques risquent probablement à long terme d'entraîner un remodelage bronchopulmonaire irréversible (bronchopathie chronique). D'où l'utilité de consulter en pneumologie...

\section{Amélioration et prévention du système respiratoire}

\subsection{Intérêt d'un entraînement spécifique des muscles respiratoires?}

Quelques travaux scientifiques récents évoquent qu'une fatigue spécifique des muscles respiratoires apparaît au décours d'efforts prolongés et pourrait participer à la diminution de la performance aérobie en réduisant l'apport en oxygène vers la périphérie et en augmentant l'inconfort respiratoire. Des travaux de physiologie suggèrent par ailleurs que lorsque les muscles respiratoires sont fatigués, une partie plus importante du débit sanguin total est déviée des muscles locomoteurs vers les muscles respiratoires [10]. Ainsi, la fatigue des muscles respiratoires pourrait précipiter le développement de la fatigue des muscles locomoteurs. D'où l'idée d'entraîner les muscles respiratoires ... Un entraînement spécifique de ces muscles peut améliorer leur efficience (capacité oxydative, nombre et taille des mitochondries, ...), retarder leur fatigue et, par conséquent, réduire la compétition du flux sanguin entre muscles respiratoires et muscles locomoteurs à l'exercice intense. Cela pourrait favoriser l'apport et l'utilisation de l' $\mathrm{O}_{2}$ par les muscles locomoteurs et ainsi augmenter les performances aérobies des sportifs. Il existe deux grandes méthodes d'entraînement des muscles respiratoires : soit un entraînement contre résistance, soit un entraînement en endurance. Cette deuxième modalité paraît la plus adéquate car elle reproduit plus fidèlement les conditions de travail de la musculature respiratoire à l'effort. Cet entraînement en endurance des muscles respiratoires est facile à mettre en place : il consiste à effectuer 30 minutes d'hyperventilation à $60 \%$ de la ventilation maximale volontaire par jour, 3 à 5 fois par semaine (appareil de type Spirotiger). Comme pour tout entraînement, la charge de travail devra augmenter progressivement au fil des semaines.

Un entraînement spécifique des muscles respiratoires peut donc être envisagé afin d'augmenter leur force et/ou endurance. De façon associée, un certain nombre d'études ont montré spécifiquement une amélioration de la tolérance à l'effort (diminution de la lactatémie, augmentation du temps de maintien d'un effort sousmaximal. ..) suite à ce type d'entrainement ; l'impact précis sur la réponse à l'exercice du sportif reste cependant encore à étayer et clarifier [11].

\subsection{Respirer moins, respirer mieux?}

Les méthodes d'entraînement consistant à limiter le débit ventilatoire se sont développées après les jeux olympiques de Mexico (1968) ; l'objectif était de reproduire des adaptations semblables à celles qui peuvent éventuellement être obtenues après un entraînement en altitude (amélioration du transport de l'oxygène jusqu'aux cellules musculaires, amélioration de l'activité des enzymes impliqués dans la production d'énergie, ....). Au cours des 10 dernières années, les propositions d'entraînement hypoxique ont évolué. Dans la phase initiale, il s'agissait de se soumettre à une hypoxie continue (vivre et s'entraîner en altitude) puis est apparu le concept d'hypoxie intermittente. Sur le plan scientifique, on s'est effectivement rendu compte que l'alternance entre les phases d'hypoxie et de normoxie était probablement plus efficace que la durée du temps d'hypoxie. Sur le plan physiologique, le fait d'arrêter de respirer pendant 20 à 30 secondes suffit à abaisser le taux de saturation en oxygène autour de $90 \%$. Cette désaturation artérielle en oxygène peut favoriser le relargage de l'oxygène (effet Bohr) et donc améliorer l'oxygénation du tissu musculaire en activité [12,13]. Cette baisse du taux d'oxygène dans le sang est également susceptible de stimuler la production d'érythropoiétine endogène. Des travaux scientifiques ont effectivement montré que retenir sa respiration quelques secondes 8 à 10 fois durant un exercice d'endurance permet d'augmenter le taux d'érythropoïétine endogène. D'autres adaptations physiologiques ont également été décrites, comme une amélioration de la régulation du $\mathrm{pH}$ et du pouvoir tampon musculaire. La méthodologie d'entraînement se décline selon différentes modalités en fonction de la discipline sportive (sports d'endurance, sports d'équipe avec ballon, natation, 400 ou 800 mètres, cyclisme sur piste...).

\subsection{Bronchomotricité et conseils à prodiguer}

Tout sportif présentant des difficultés respiratoires à l'effort doit bénéficier d'un bilan pneumologique. En effet, les plaintes bronchitiques sont de plus en plus fréquentes chez les sportifs impliqués dans des activités soutenues d'endurance et pourraient évoluer vers la chronicité si l'on n'y prend pas garde. Parmi les conseils pratiques à prodiguer chez ces sportifs, il est utile de rappeler l'intérêt majeur de maintenir, chaque fois que possible durant l'effort, une respiration nasale. La respiration par le nez, en effet, modifie de façon très efficace les caractéristiques de l'air inspiré si bien que, même dans des conditions extrêmement basses de température et d'humidité ambiante, l'air qui atteint le nasopharynx est humidifié à près de 100 \% d'humidité relative avec une température aux environs de 32 à 35 degrés. Donc, un sportif " asthmatique » qui respire par le nez au décours d'un effort physique atténue voire abolit les effets néfastes de l'air froid et de la sécheresse ambiante.

Certaines activités physiques sont également plus inductrices de bronchospasme à l'effort que d'autres. Par exemple, parmi les activités de terrain, il est préférable de recommander des activités physiques d'intensité faible à modérée, ou pratiquées de manière intermittente, moins inductrices d'asthme que des activités prolongées, continues et d'intensité soutenue. L'intérêt de l'échauffement comme moyen de prévention du bronchospasme à l'effort est une notion qui, sur le plan scientifique, reste controversée. Par contre, le retour au calme sur une période de 10 à 15 minutes aurait des effets bénéfiques en limitant les changements brusques de température dans la muqueuse des voies aériennes. L'hydratation régulière du sportif asthmatique, en réduisant l'assèchement des bronches, diminue également les complications bronchiques. Enfin, tout sportif présentant des plaintes bronchospastiques à l'effort doit avoir, à portée de main, un médicament d'urgence 
(en particulier les bêta-2-mimétiques qui permettront d'éviter l'apparition des symptômes de l'asthme quasi dans 90 \% des cas).

\section{Conclusion}

Chez le sportif sain, le poumon a d'énormes réserves au repos, ce qui lui permet de faire face à l'augmentation importante des besoins d'échanges gazeux lors d'un effort physique soutenu. De nombreuses fonctions du système respiratoire se modifient en réponse à l'exercice. Ce système n'est généralement pas considéré comme un facteur limitant de la performance. Ainsi, lors d'une préparation physique en endurance, le système respiratoire est le siège d'adaptations spécifiques qui améliorent encore son efficacité. Cependant, dans certaines circonstances, chez les sportifs très entraînés en endurance, peuvent apparaître des anomalies dans le fonctionnement du système respiratoire, reflétant probablement l'atteinte d'une limite d'adaptation à la préparation athlétique.

\section{Déclaration de liens d'intérêts}

Les auteurs n'ont pas précisé leurs éventuels liens d'intérêts.

\section{Références}

[1] Réponses ventilatoires à l'exercice aigu.Kenney WL, Wilmore JM, Costill DL, editors. Physiologie du sport et de l'exercice. Bruxelles: De Boeck University; 2013. p. $186-92$
[2] Anderson GS, Rhodes EC. A review of blood lactate and ventilatory methods of detecting transition thresholds. Sports Med 1989;8:43-5.

[3] Kenny WL, Wilmore JH, Costill DL, editors. Adaptation to aerobic and anaerobic training. Physiology of sport and exercise. Leeds: Human Kinetics; 2015. p. 261-91.

[4] Powers S, Criswell D, Fu-Kong L, Dodd S, Silverman M. Diaphragmatic fiber type specific adaptation to endurance exercise. Respir Physiol 1992;89: 195-207.

[5] Ma JL, Dutch MJ. Extreme sports: extreme physiology. Exercise induces pulmonary oedema. Emerg Med Australas 2013;25:368-71.

[6] Zavorsky GS, Milne EN, Lavorini F, Rienzi JP, Lavin KM, Straub AM, et al. Interstitial lung edema triggered by marathon running. Respir Physiol Neurobiol 2014;190:137-41.

[7] Préparation physique et appareil respiratoire.Koechlin-Ramonatxo C, Prefaut C, editors. La preparation physique. Paris: Masson; 2007. p. 177-82.

[8] Helenius IJ, Tikkanen HO, Haahtela T. Occurrence of exercise induced bronchospasm in elite runners: dependence on atopy and exposure to cold air and pollen. Br J Sports Med 1998;32:125-9.

[9] Karlese KH, Kowalski ML. Asthma, allergy, the athlete and the Olympics. Allergy 2008;63:383-6.

[10] Hajghanbari B, Yamabayashi C, Buna T, Coelho J, Freedman KD, Morton $\mathrm{TA}$, et al. Effects of respiratory muscle training on performance in athletes: a systematic review with meta-analyses. J Strength Cond Res 2013;27: 1643-63.

[11] Mickleborough TD, Nichols T, Lindley MR, Chatham K, Ionescu AA. Inspiratory flow resistive loading improves respiratory muscle function and endurance capacity in recreational runners. Scand J Med Sci Sports 2010;20: 458-68.

[12] Faiss R, Girard O, Millet GP. Advancing hypoxic training in team sports: from intermittent hypoxic training to repeated sprint training in hypoxic. Br J Sports Med 2013;47(Suppl. 1):i45-50.

13] Casey DP, Joyner MJ. Compensatory vasodilatation during hypoxic exercise: mechanisms responsible for matching oxygen supply to demand. J Physiol 2012;590:6321-6. 